\title{
Partial Decentralized Wireless Control Through Distributed Computing for Seismically Excited Civil Structures: Theory and Validation
}

\author{
R. Andrew Swartz and Jerome P. Lynch, Member, IEEE
}

\begin{abstract}
This study presents a partially-decentralized control scheme for wireless control of large civil structures. Reducing the seismic response of a structure reduces the likelihood of significant damage to the structure or its occupants. The cost of implementing such a system is reduced by employing wireless sensors, thus avoiding the prohibitive cost of installing cable-based systems. Wireless sensors with embedded computational abilities capable of sensing and actuation can perform structural control tasks without a central computer. A wireless sensor network is developed composed of wireless sensors employing identical static Kalman estimators. The wireless sensors command actuators with LQR derived state-feedback control forces based on state vectors locally calculated from embedded Kalman estimators. Each unit compares the estimated state values to their local measured state data; in the case where the estimate error exceeds a predefined threshold, replaces the estimated value with the measured value and transmits the update to the entire wireless sensor network. By strategically transmitting only some of the measured data, this scheme reduces demand on the limited wireless bandwidth and energy resources associated with wireless sensor networks. Numerical simulation results using this scheme are presented as well as experimental results for a half-scale 3-story steel structure with magnetorheological (MR) dampers controlling each floor.
\end{abstract}

\section{INTRODUCTION}

$\mathrm{L}_{\mathrm{a}}^{\mathrm{a}}$ arge civil structures (e.g. buildings and bridges) present a unique set of challenges for structural control. Due to their mass, the control forces often associated with civil seismic disturbance rejection are exceedingly large. Furthermore, the size of civil structures render the cost and effort of running wires between sensors, actuators and controllers become prohibitive. Several studies reported in the literature have applied semi-active control schemes to the first problem [1-4]. The second problem can be overcome by the use of wireless sensors for sensing and control [5-7]. As demonstrated by Straser and Kiremidjian [8] and Lynch, et al. [9], wireless sensors with embedded

Manuscript received September 15, 2006.

$\mathrm{R}$. Andrew Swartz is a graduate student with the University of Michigan, Department of Civil and Environmental Engineering, Ann Arbor, MI 48109-2125 USA.(e-mail: asgard@umich.edu).

J. P. Lynch is an Assistant Professor with the University of Michigan, Department of Civil and Environmental Engineering and the Department of Electrical Engineering and Computer Science, Ann Arbor, MI 48109-2125 USA. (phone: 734-615-5290; fax: 734-764-4292; e-mail: jerlynch@umich.edu). computational abilities can save energy and bandwidth by eliminating the need to transmit raw data in its entirety. In previous work, Wang, et al. [10] demonstrated the use of wireless sensors for structural control using a fullycentralized control scheme with all data wirelessly transmitted between units, thereby validating the effectiveness of wireless control for civil structures. The fully-centralized method requires a large amount of bandwidth and battery energy to transmit data, both scarce system resources.

In a novel partially-decentralized control scheme first proposed by Yook, et al. [11], bandwidth is traded for control performance in a networked (wired or wireless) control system. By selectively sharing data between controllers, improvements can be made over the performance of a fully-decentralized control scheme while utilizing less bandwidth and communications energy than the fully-centralized solution. In this scheme, identical static Kalman estimators are embedded in multiple, networked controllers. Each unit calculates an estimate of the full state from locally available measured plant outputs. The units compare the estimated state value at their location to the corresponding measured data. If they differ by more than a predetermined threshold value, the controllers then replace the estimated value with the measured value in the state vector. The state update is then broadcast to the networked controllers, thereby keeping the state estimate the same throughout the system. This control scheme has been explored for large civil structures previously using numerical simulations [12].

In this study, wireless sensors commanding co-located actuators, employ both Kalman estimators and state value recovery to control a 3-story, half-scale, steel structure actuated by MR-dampers on each floor. In developing the control scheme, the state-space model is developed, followed by the derivations of the LQR control gains and static Kalman estimators. Next, results of a numerical simulation study are presented with control cost functions used as performance metrics. Finally, experimental results are presented demonstrating the change in control performance and bandwidth utilization as the error threshold changes.

\section{PARTIALLY-DECENTRALIZED CONTROLER}

This section presents the structural control scheme using the redundant static Kalman state estimation and recovery algorithm running on independent wireless sensors 
assembled as a networked control system [11]. First, the state-space formulation for a lumped mass shear structure based on interstory drift is developed. Next, a linearquadratic regulator (LQR) optimal state-feedback control gain is derived for the system. Finally, the Kalman estimator for each sensing unit is formulated.

\section{A. State-Space System Model}

The control solution and the Kalman estimator are based on a linear, time-invariant system model posed in statespace. The state-space model formulation begins with the matrix equation of motion of the structure:

$$
\mathbf{M} \ddot{\mathbf{z}}(t)+\mathbf{C}^{\prime} \dot{\mathbf{z}}(t)+\mathbf{K z}(t)=\mathbf{u}(t)+\mathbf{p}(t)
$$

where $\mathbf{M}, \mathbf{C}^{\prime}$ and $\mathbf{K}$ are the mass, damping and stiffness matrices, respectively, $\mathbf{z}$ is the vector of lateral response, $\mathbf{u}$ is the control force, and $\mathbf{p}$ is the seismic disturbance based on ground motion. In order to allow the individual wireless sensors to directly measure their local state variable, the state-space model of the system is based on interstory drift and interstory velocity at each floor rather than traditional displacement and velocity relative to the ground. The mass matrix contains non-diagonal terms to account for interstory acceleration values in the force equilibrium equations. Formulated for an $n$-degree-of-freedom structure, $\mathbf{M}$ is:

$$
M=\left[\begin{array}{ccccc}
m_{1} & 0 & \cdots & & 0 \\
m_{2} & m_{2} & & & \\
\vdots & & \ddots & & \vdots \\
m_{n-1} & m_{n-1} & & m_{n-1} & 0 \\
m_{n} & m_{n} & \cdots & m_{n} & m_{n}
\end{array}\right]
$$

Stiffness and damping matrices for such a structure are:

$$
\mathbf{K}=\left[\begin{array}{ccccc}
k_{1} & -k_{2} & 0 & & 0 \\
0 & k_{2} & -k_{3} & \cdots & 0 \\
0 & 0 & k_{3} & & 0 \\
& \vdots & & \ddots & \\
0 & 0 & 0 & & k_{n}
\end{array}\right] \quad \mathbf{C}=\left[\begin{array}{ccccc}
c_{1} & -c_{2} & 0 & & 0 \\
0 & c_{2} & -c_{3} & \cdots & 0 \\
0 & 0 & c_{3} & & 0 \\
& \vdots & & \ddots & \\
0 & 0 & 0 & & c_{n}
\end{array}\right]
$$

In state-space, the system model is:

$$
\dot{\mathbf{x}}=\mathbf{A x}+\mathbf{B u}+\mathbf{E p}
$$

where

$$
\mathbf{A}=\left[\begin{array}{cc}
\mathbf{0} & \mathbf{I} \\
-\mathbf{M}^{-1} \mathbf{K} & -\mathbf{M}^{-1} \mathbf{C}
\end{array}\right] \quad \mathbf{B}=\left[\begin{array}{c}
\mathbf{0} \\
-\mathbf{M}^{-1}
\end{array}\right] \quad \mathbf{E}=\left[\begin{array}{l}
\mathbf{0} \\
\mathbf{1}
\end{array}\right]
$$

$\mathbf{x}$ is the state vector of interstory drift and interstory velocity values, and $\mathbf{u}$ is the control force. The output at the $i^{\text {th }}$ degree of freedom (DOF) is the velocity difference between the $i$ and $i-1 \mathrm{DOF}$. This interstory velocity can be measured using absolute velocity meters located on floors above and below the $i^{\text {th }}$ controller. Because of the practical difficulties in obtaining an accurate, direct measure of interstory drift, the drift is not included in the output. The system output is then:

$$
\mathbf{y}=\mathbf{C x}+\mathbf{D u}+\mathbf{F p}
$$

where

$$
\mathbf{C}=\left[\begin{array}{ll}
\mathbf{0} & \mathbf{I}
\end{array}\right] \quad \mathbf{D}=[\mathbf{0}] \quad \mathbf{F}=[\mathbf{0}]
$$

and $\mathbf{y}$ is the measured interstory velocity.

\section{B. Optimal LQR Controller Design}

A linear quadratic regulator (LQR) is selected to provide the optimum control performance for the available control force. For this method, a gain matrix, $\mathbf{G}$, is derived such that the optimal control force is proportional to the state:

$$
\mathbf{u}=-\mathbf{G z}
$$

where $\mathbf{u}$ minimizes the cost function:

$$
J(\mathbf{u})=\sum_{n=1}^{\infty}\left(\mathbf{x}^{\mathrm{T}}[n] \mathbf{Q} \mathbf{x}[n]+\mathbf{u}^{\mathrm{T}}[n] \mathbf{R} \mathbf{u}[n]\right) .
$$

The matrices $\mathbf{Q}$ and $\mathbf{R}$ in (8) are positive definite and weight the emphasis that the control solution will place on minimizing both the response of the system and the control input required by the system, respectively. Larger values within $\mathbf{Q}$ relative to the corresponding values in $\mathbf{R}$ will result in greater control force used to achieve the control objective.

Care must be taken in selecting a relative weighting between $\mathbf{Q}$ and $\mathbf{R}$. Although semi-active devices (like MRdampers) render the system inherently stable (in a bounded input-bounded output sense), actuators are limited by maximum and sometimes minimum control forces. Clearly, an implicit assumption of the LQR derived gain is that the actuators are capable of applying the force desired. The final $\mathbf{Q}$ and $\mathbf{R}$ are selected such that the maximum permissible control force of a $20-\mathrm{kN}$ MR is generally not exceeded by a strong earthquake motion.

As damage to structural components (e.g. columns) is often the result of excessive interstory drift, $\mathbf{Q}$ and $\mathbf{R}$ are selected in a manner that minimizes drift while employing attainable control forces. Weighting is also given to minimizing interstory velocity as a means of removing the kinetic energy from the system. This can be an effective way to reduce the total displacement that the structure experiences during a seismic event. The $\mathbf{Q}$ matrix is specified as:

$$
\mathbf{Q}=\left[\begin{array}{cc}
\mathbf{I} & 10 \mathbf{I} \\
10 \mathbf{I} & 100 \mathbf{I}
\end{array}\right]
$$

The $\mathbf{R}$ matrix is simply:

$$
\mathbf{R}=10^{-9}[\mathbf{I}]
$$

Having selected $\mathbf{R}$ and $\mathbf{Q}$, the discrete-time control gain matrix, $\mathbf{G}$, is calculated from:

$$
\mathbf{G}=\left(\mathbf{B}_{\mathrm{d}}{ }^{\mathrm{T}} \mathbf{S B}_{\mathrm{d}}+\mathbf{R}\right)^{-1}\left(\mathbf{B}_{\mathrm{d}}{ }^{\mathrm{T}} \mathbf{S} \mathbf{A}_{\mathrm{d}}\right)
$$

where $\mathbf{A}_{\mathbf{d}}$ and $\mathbf{B}_{\mathbf{d}}$ are the discrete-time, zero-order hold approximations of $\mathbf{A}$ and $\mathbf{B}$, and $\mathbf{S}$ is the solution of the Riccati equation [13]:

$$
\mathbf{A}_{\mathbf{d}}{ }^{\mathrm{T}} \mathbf{S} \mathbf{A}_{\mathbf{d}}-\mathbf{S}-\left(\mathbf{A}_{\mathbf{d}}{ }^{\mathrm{T}} \mathbf{S B}_{\mathrm{d}}\right)\left(\mathbf{B}_{\mathrm{d}}^{\mathrm{T}} \mathbf{S B}_{\mathbf{d}}+\mathbf{R}\right)^{-1}\left(\mathbf{B}_{\mathrm{d}}^{\mathrm{T}} \mathbf{S} \mathbf{A}_{\mathbf{d}}\right)+\mathbf{Q}=0
$$




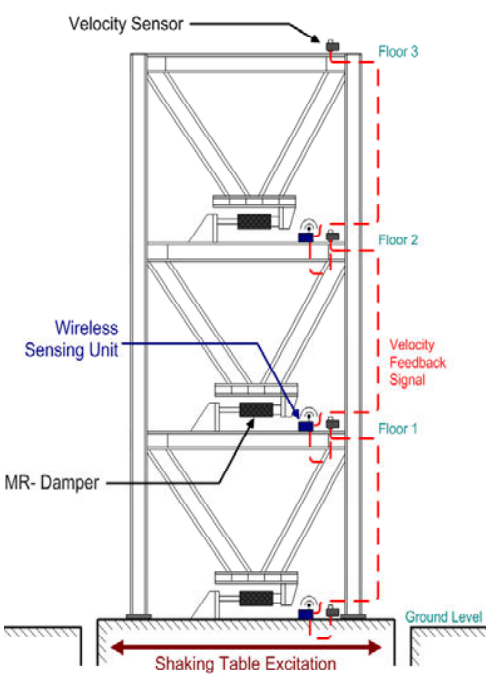

Figure 1. NCREE Structure

\section{Kalman Estimation}

Control forces at each wireless sensor are calculated from the complete state matrix despite the fact that individual wireless sensors only measure a small portion of the state. To compensate for this fact, each wireless sensing unit is programmed with an embedded Kalman derived state estimator for which the measured output is the local interstory velocity. The static Kalman estimator calculates an estimate of the state vector at the next time step based on the state vector estimate from the current step and the measured output by:

$$
\mathbf{x}[n+1]=\mathbf{A}_{\text {est }} \mathbf{x}[n]+\mathbf{B}_{\text {est }} \mathbf{y}[n]
$$

where:

$$
\mathbf{A}_{\text {est }}=\left(\mathbf{A}_{\mathrm{d}}-\mathbf{B}_{\mathrm{d}} \mathbf{G}-\mathbf{L C}_{\mathrm{d}}+\mathbf{L D G}\right) \quad \mathbf{B}_{\mathrm{est}}=\mathbf{L}
$$

$\mathbf{L}$ is the Kalman estimation gain matrix, and $\mathbf{C}_{\mathbf{d}}$ is the zeroorder hold augmented, discrete-time $\mathbf{C}$ matrix.

The wireless sensors can however, take advantage of their wireless communication channel to share information. Each wireless sensor compares the measured state information that is available to it locally (interstory velocity) to the estimated value. If those values differ by a predetermined error threshold, $E$, the unit will replace the estimated value in the local state vector with the measured value. Additionally, the unit will broadcast its measured value for the rest of the network to use. This communication between units keeps the state vector estimation error consistent throughout the network. Improved velocity estimates help reduce the error in estimated drift as small errors in velocity estimates, uncorrected over time, can lead to large errors in estimated drift. An error threshold value of zero will result in transmission of every interstory velocity value for every time step, effectively becoming a fullycentralized solution at the cost of considerable bandwidth and energy consumption. An error threshold so large that it is never exceeded (e.g. infinity) results in no communication between wireless units, effectively a fully-decentralized solution with the cost of lower estimator accuracy and degraded controller performance. A spectrum of threshold values between zero and infinity are explored to demonstrate a partially-decentralized wireless control solution. A reasonable tradeoff between estimator error and communication overhead can be achieved by judiciously selecting the error threshold. Stability of such a method has been investigated previously by Yook, et al [11].

\section{NUMERICAL EXAMPLE}

A numerical model is analyzed in MATLAB to demonstrate the distributed Kalman estimation technique. The model is based on a 3-story steel structure located at the National Center for Research in Earthquake Engineering (NCREE), Taipei, Taiwan that will be employed during the experimental phase of the project (Figure 1). The structure, $9 \mathrm{~m}$ tall, is constructed on a $5 \times 5 \mathrm{~m}^{2}$ square shaking table capable of actuation in six DOF. The seismic mass of each floor is $6000 \mathrm{~kg}$. The interstory stiffness is taken as $1.69 \times 10^{6} \mathrm{~N} / \mathrm{m}, 1.95 \times 10^{6} \mathrm{~N} / \mathrm{m}$, and $1.60 \times 10^{6} \mathrm{~N} / \mathrm{m}$ for floors 1,2 , and 3 respectively. The equivalent viscous damping values for floors 1,2 , and 3 are taken to be $5.561 \times 10^{6}$ $\mathrm{N} /(\mathrm{m} / \mathrm{s}), \quad 3.693 \times 10^{6} \quad \mathrm{~N} /(\mathrm{m} / \mathrm{s})$, and $0.890 \times 10^{6} \quad \mathrm{~N} /(\mathrm{m} / \mathrm{s})$ respectively. A $20-\mathrm{kN}$ MR damper is installed upon each floor of the structure using a steel V-brace configuration. The damping coefficient of the MR damper can be varied in real-time to mitigate the lateral response of the structure to ground motion.

One wireless sensor is installed upon each floor of the structure, but not on the roof; in total, three wireless sensors are installed. The role of the wireless sensor is to megsure the interstory velocity of the floor, calculate an optimal control force, apply a voltage command signal to the damper, and wirelessly exchange data with other wireless sensors. To determine interstory velocity, each wireless sensor measures the absolute velocity from a velocity meter installed upon the floor above and upon the floor the wireless sensor is installed. In the numerical simulation, white noise is added to each velocity measurement consistent with the specified noise of the velocity meter to be used during the experimental phase of the study (Tokyo Sokushin VSE-15D velocity meter whose root mean square noise is specified as $0.5 \mathrm{~mm} / \mathrm{s}$ ). Wireless packet loss is modeled as a random process with approximately $2.0 \%$ of packets dropped by the intended receiving wireless sensor; this drop rate is based upon a previous validation study of wireless sensors for real-time control conducted by Wang, et al. [10]. 

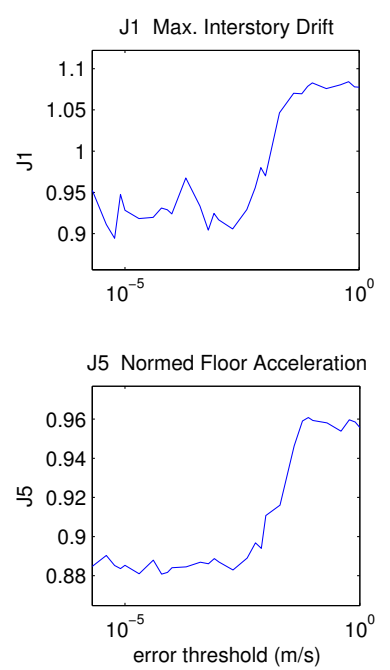
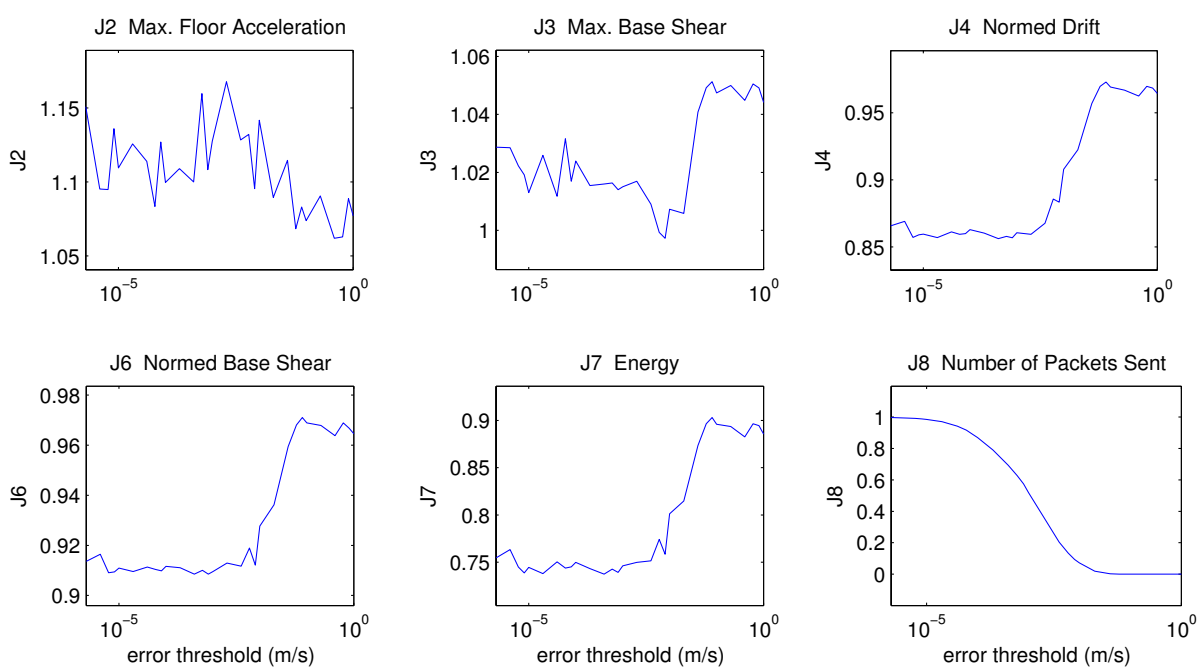

Figure 2. Numerical example cost function results for El Centro, $0.100 \mathrm{~m} / \mathrm{s}^{2}$ (100 gal).

The model is subjected to four different earthquake ground acceleration records: El Centro NS 1940 (NS-USGS Station 117), Loma Prieta NS 1989 (Palo Alto SLAC Lab), Kobe EW 1995 (JMA Station), and Sylmar NS 1971 (NSCDMG Station 24514). The records are normalized by absolute maximum acceleration to $0.050 \mathrm{~m} / \mathrm{s}^{2}$ (50 gals), $0.100 \mathrm{~m} / \mathrm{s}^{2}(100$ gals $), 0.150 \mathrm{~m} / \mathrm{s}^{2}(150$ gals $)$, and $0.200 \mathrm{~m} / \mathrm{s}^{2}$ (200 gals). The structural response for each acceleration record/peak acceleration combination is recorded and normalized to the response of the uncontrolled structure for the same acceleration record/peak acceleration combination using a number of cost functions described below.

To assess the performance of the control scheme, eight cost functions are proposed; the first six, J1-J6, are based on cost functions developed in [14]. $J 1$ measures peak interstory drift, $d$, (over all floors and all time) normalized to the peak interstory drift for the uncontrolled structure:

$$
J 1=\frac{\max _{\text {Floor }, t}\left(\left|d_{\text {controlled }}\right|\right)}{\max _{\text {Floor }, t}\left(\left|d_{\text {uncontroll ed }}\right|\right)}
$$

Similarly $J 2$ measures normalized peak acceleration and $J 3$ measures normalized peak base shear:

$$
\begin{aligned}
& J 2=\frac{\max _{\text {Floor }, t}\left(\left|\ddot{z}_{\text {controlled }}\right|\right)}{\operatorname{mlloor}, t_{t}\left(\left|\ddot{z}_{\text {uncontrolled }}\right|\right)} \\
& J 3=\frac{\max \left(\left|\ddot{\mathbf{z}}_{\text {controlled }} \mathbf{W}\right|\right)}{\max \left(\left|\ddot{\mathbf{z}}_{\text {uncontrolled }} \mathbf{W}\right|\right)}
\end{aligned}
$$

where $\mathbf{W}$ is the seismic mass vector. To measure the performance of the controller over the entirety of the record, $J 4, J 5$, and $J 6$ measure the normalized norms of the drifts vector, $\mathbf{d}$, acceleration vector, and base shears, respectively:

$$
\begin{aligned}
& J 4=\frac{\operatorname{norm}\left(\left|\mathbf{d}_{\text {controlled }}\right|\right)}{\operatorname{norm}\left(\left|\mathbf{d}_{\text {uncontrolled }}\right|\right)} \\
& J 5=\frac{\operatorname{norm}\left(\left|\ddot{\mathbf{z}}_{\text {controlled }}\right|\right)}{\operatorname{norm}\left(\left|\ddot{\mathbf{z}}_{\text {uncontrolled }}\right|\right)}
\end{aligned}
$$

$$
J 6=\frac{\operatorname{norm}\left(\left|\ddot{\mathbf{z}}_{\text {controlled }} \mathbf{W}\right|\right)}{\operatorname{norm}\left(\left|\ddot{\mathbf{z}}_{\text {uncontrolled }} \mathbf{W}\right|\right)} \cdot
$$

A measure of the kinetic energy within the system over the system's full trajectory $\left(t \in\left(0, t_{f}\right)\right)$ is obtained from $J 7$ :

$$
J 7=\frac{\left[\frac{1}{N} \sum_{i=1}^{N} \mathbf{x}_{i}^{\mathrm{T}}\left[\begin{array}{cc}
\mathbf{K} & \mathbf{0} \\
\mathbf{0} & \mathbf{M}
\end{array}\right]_{\mathbf{x}_{i}}\right]_{\text {controlled }}}{\left[\frac{1}{N} \sum_{i=1}^{N} \mathbf{x}_{i}^{\mathrm{T}}\left[\begin{array}{cc}
\mathbf{K} & \mathbf{0} \\
\mathbf{0} & \mathbf{M}
\end{array}\right] \mathbf{x}_{i}\right]_{\text {uncontrolled }}}
$$

A measure of the bandwidth demand for the wireless units is obtained from $J 8$ :

$$
J 8=\frac{(\# \text { Data Transmissi ons Sent })}{(\# \text { Time Steps })(\# \text { Wireless Units })}
$$

Figures 2 shows these cost functions as a function of error threshold for El Centro, $0.100 \mathrm{~m} / \mathrm{s}^{2}$ (100 gal). Again, exceeding the error threshold triggers updating via wireless communication.

As can be seen in Figure 2, the majority of the cost indices follow the general trend of low cost indices at low error thresholds with increasing indices as the error threshold is increased. This trend is consistent with the system behaving more like a decentralized system as the error threshold is increased. Cost index $J 2$ does not seem to follow the trend anticipated; this could be due in part to the LQR solution formulated to specifically mitigate the velocity and displacement response of the structure (as opposed to explicitly attempting to minimize accelerations). Cost index $J 8$ illustrates the reduction in the use of the wireless communication channel as the error threshold is increased.

\section{EXPERIMENTAL RESULTS}

Validation of the technique is performed on the 3-story NCREE steel structure using the University of Michigan "Narada" wireless sensor (Figure 3a). The Narada wireless sensor features a low-voltage, 8-bit Atmel ATmega 128 microcontroller computational core with $128 \mathrm{kB}$ of external 


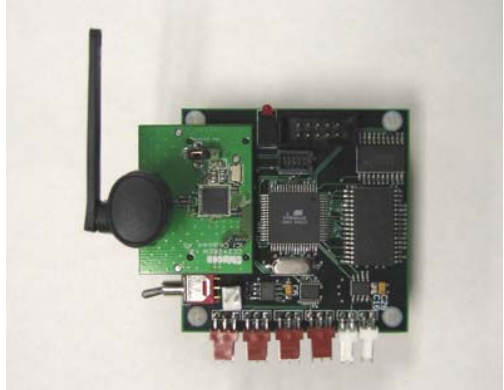

(a)

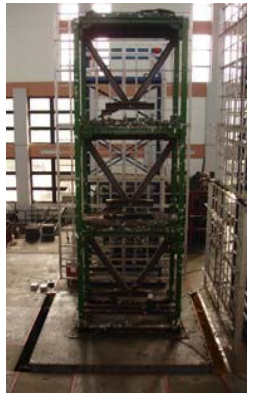

(b)
Figure 3. (a) Narada wireless sensor; (b) NCREE test structure.
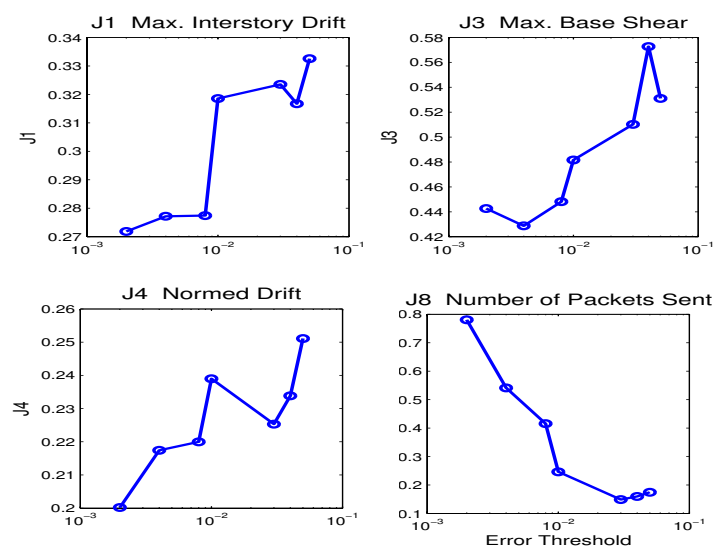

Figure 4. Cost functions (EI Centro 100 gal).

SRAM. The sensing interface consists of a 16-bit, 4channel, Texas Instruments ADS8341 analog-to-digital converter (ADC). The actuation interface is a 12-bit, 2channel, Texas Instruments DAC7612 digital-to-analog converter (DAC). Communication between Narada units is accomplished using a Chipcon 2420, IEEE 802.15.4 (Zigbee) compliant radio. The Zigbee physical layer is left intact for the control experiments. The Zigbee medium access control (MAC) layer however, is deemed too slow for control applications with single packet transmissions requiring approximately $16 \mu \mathrm{s}$. The control experiment instead utilizes a simplified MAC layer that provides a time window for each wireless sensor to broadcast data. Packet receipt acknowledgement is also removed to increase speed of transmission. With these changes, packets can be transmitted in approximately $1.2 \mu \mathrm{s}$.

To provide the control force, MR dampers are installed in the NCREE structure at the shaking table level and on floors 1 and 2. MR-dampers are semi-active control devices that change their damping force based on the current provided to them. MR dampers exhibit hysteresis, as characterized by [2]. To generate the desired control force, a modified Bouc-Wen model is employed. Given the state of the damper and the interstory velocity, the potential control forces achievable from a finite set of MR command voltages between 0 and $1.2 \mathrm{~V}$ (to be output from the actuation interface of the Narada unit) is computed. The voltage that produces the force nearest the desired control force is selected as the output voltage. Upon determination of the control force and MR damper voltage, the Narada wireless sensor issues the voltage using the actuation interface. Tokyo Sokushin VSE-15D velocity meters provide absolute velocity measurements from which the wireless sensor can calculate interstory velocities. Two channels are employed to measure velocity above and below the controllers.

The semi-active, partially-distributed, wireless control scheme is tested on the NCREE structure with El Centro, $0.100 \mathrm{~m} / \mathrm{s}^{2}$ (100 gal), ground acceleration excitation. Tests are performed for a range of error thresholds in terms of interstory velocity. The error thresholds utilized are $[0$, 0.002, 0.004, $0.0060 .008,0.010,0.020,0.030,0.040,0.050$, $0.060,0.5000] \mathrm{m} / \mathrm{s}$. The sampling, control, and estimation step period, $T_{s}$, used is $40 \mathrm{~ms}$ to allow time for sampling, computation, communication, as well as additional time for resolution of communication errors (occasional packet collisions), resulting in a sampling rate of $25 \mathrm{~Hz}$. Cost functions for drift and bandwidth $(J 1, J 3, J 4$, and $J 8)$ versus error threshold are shown in Figure 4 showing the trend of decreasing control performance and decreasing bandwidth usage as the error threshold is increased. Cost functions in Figure 4 are normalized to the response of the bare structure without MR-dampers connected. A brief example is presented to illustrate, in absolute terms, the number of wireless updates utilized. When $E$ is set to $0.006 \mathrm{~m} / \mathrm{s}, J 8$ is 0.4808. This value corresponds to 2524 packets transmissions by three units (roughly, evenly divided) over the course of a $70 \mathrm{~s}$ test. Figure 5 shows maximum interstory drift versus floor number for the various error thresholds including no control and passive control cases. In Figure 6, maximum drift values are presented versus error threshold for each floor. Generally, the maximum drift trends upward as error threshold increases with the exception of floor 3 .

\section{CONCLUSIONS}

By transmitting measured state data only when it exceeds a given error threshold, the wireless sensors can save scarce communication bandwidth and energy resources by limiting their use of their wireless modems. This is accomplished by leveraging the on-board computational abilities of the wireless sensors. As that error threshold decreases, the control performance approaches that of a fully-centralized solution and the number of data packets required increases. By increasing the threshold, the control performance increases to that of a fully-decentralized solution and the number of data packets required decreases. Experimental data confirms the trend of decreasing control performance and decreasing bandwidth utilization as the error threshold increases.

This control scheme overcomes some of the limitations of wireless control. The effects of delayed or dropped packets are mitigated by the Kalman estimation. The estimated state values ensure that a control force calculation will be available for every control step. If a unit misses a packet at least, the estimated value would still be available. 


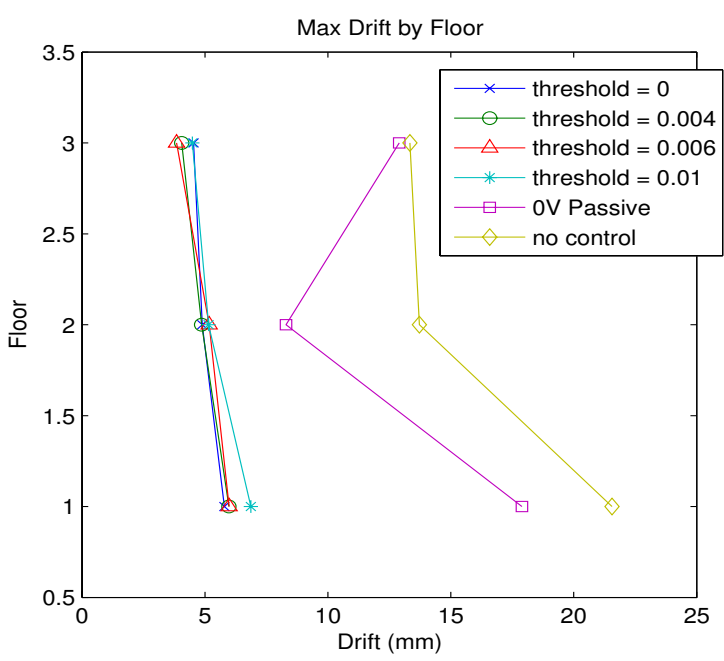

Figure 5. Experimental max drift versus floor.

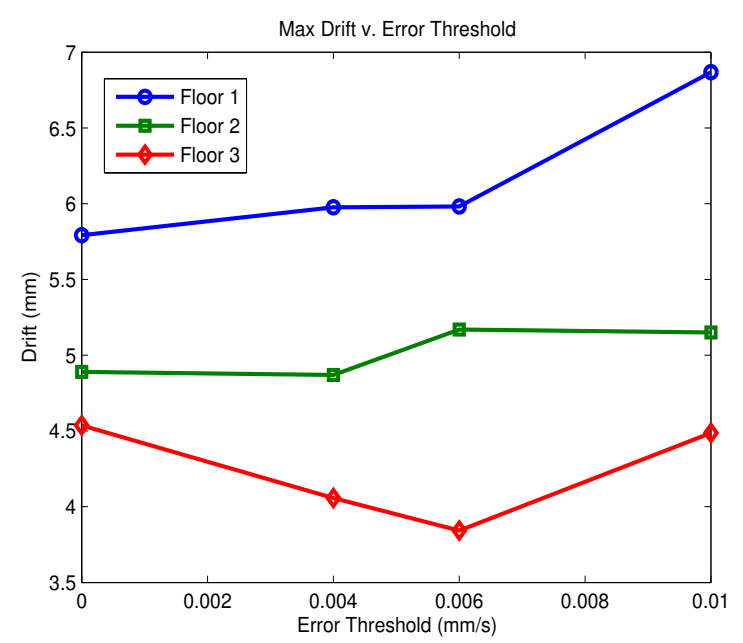

Figure 6. Experimental max drift versus error threshold.
Communication problems reduce the sampling rate achievable for the experimental setup and hence, the effectiveness of the control algorithm. While $40 \mathrm{~Hz}$ sampling time is possible for the Narada unit with this control scheme, $25 \mathrm{~Hz}$ is used to allow extra time to clear communication errors due to packet collisions with other wireless networks within the $2.4 \mathrm{GHz}$ industrial, scientific, and medical (ISM) band including IEEE 802.11 networks and wireless equipment.

Improvements in the error handling abilities of the communications protocol will allow the obtainable sampling rate to be larger. Future work should include applications to larger sensor networks where direct communication between all sensors becomes untenable. LQR control is optimal only when the desired control force is obtainable by the controller; for the MR-damper, this was generally, but not always, true. The effectiveness of control formulations other than LQR should also be explored.

\section{ACKNOWLEDGEMENTS}

The authors would like to acknowledge their gratitude for invaluable assistance provided by Professor Kincho Law and Mr. Yang Wang of Stanford University, Professor Dawn Tilbury of The University of Michigan, and Professor ChinHsiung Loh of National Taiwan University. This research has been partially sponsored by the Office of Naval Research through the Young Investigator Program.

\section{REFERENCES}

[1] Kurata, N., T. Kobori, M. Takahashi, N. Niwa and H. Midorikawa, "Actual seismic response controlled building with semi-active damper system," Earthquake Engineering and Structural Dynamics, vol. 28, no. 11, pp. 1427-1447, 1999.

[2] Lin, P.-Y., P.N. Roschke, and C.-H. Loh, "System Identification and Real Application of a Smart Magneto-Rheological Damper," Proc. of the 2005 International Symposium on Intelligent Control, Limassol, Cypru, 2005s.

[3] Hatada T., T. Kobori, M. Ishida, N. Niwa, "Dynamic analysis of structures with Maxwell model," Earthquake Engineering and Structural Dynamics, vol. 29, no. 2, pp. 159-176, 2000.
[4] Spencer, B.F., S.J. Dyke, M.K. Sain, J.D. Carlson, "Phenomenological model for magnetorheological dampers," Journal of Engineering Mechanics, vol. 123, no. 3, pp. 230-238, 1997.

[5] Wang, Y., A. Swartz, J.P. Lynch, K.H. Law, K-C. Lu, C-H. Loh, "Wireless feedback structural control with embedded computing," Proc. of the SPIE $11^{\text {th }}$ International Symposium on Nondestructive Evaluation for Health Monitoring and Diagnostics, San Diego, CA, 2006.

[6] Ploplys N.J., P.A. Kawka, A.G., Alleyne, "Closed-loop control over wireless networks," IEEE Control Systems Magazine, pp. 58-71, 2004.

[7] Kawka, P.A., A.G. Alleyne, "Stability and Feedback Control of Wireless Networked Systems," Proceedings of the 2005 American Control Conference, pp. 2953-2959, 2004.

[8] Straser, E.G., A.S. Kiremidjian, "A Modular, Wireless Damage Monitoring System for Structures," Report No. 128, John A. Blume Earthquake Engineering Center, Department of Civil and Environmental Engineering, Stanford University, Stanford, California, 1998.

[9] Lynch, J.P., A. Sundararajan,, K.H. Law, A.S. Kiremidjian, E. Carryer, "Embedding damage detection algorithms in a wireless sensing unit for attainment of operational power efficiency," Smart Materials and Structures, IOP, 13(4): 800-810, 2004.

[10] Wang, W., R.A. Swartz, J.P. Lynch, K.H. Law, K-C. Lu and C-H. Loh "Decentralized Civil Structural Control using a Real-time Wireless Sensing and Control System," Proceedings of the 4th World Conference on Structural Control and Monitoring (4WCSCM), San Diego, CA, 2006.

[11] Yook, J.K., D.M. Tilbury, and N.R. Soparkar, "Trading computation for bandwidth: reducing communication in distributed control systems using state estimators," IEEE Transactions on Control Systems Technology, vol. 10, no. 4, pp. 503-518, 2002.

[12] Seth, S., J.P. Lynch, D.M. Tilbury, "Wirelessly networked distributed controllers for real-time control of civil structures," Proceedings of the 2005 American Control Conference, pp. 2946-2952, 2005.

[13] Stengle, R.F., Optimal Control and Estimation, Dover Publications Inc. Mineola, NY, 1994.

[14] Ohtori, Y., R. E. Christenson and B. F. Spencer, Jr., "Benchmark control problems for seismically excited nonlinear buildings," Journal of Engineering Mechanics, vol. 130, no. 4, pp. 366-385, 2004. 